\title{
GERMINACIÓN DE Ischaemum rugosum Salisb. EN RESPUESTA A ESTIMULOS AMBIENTALES Y QUÍMICOS
}

\author{
Ischaemum rugosum Salisb. GERMINATION REACTION TO \\ ENVIRONMENTAL AND CHEMICAL STIMULI
}

\author{
Alfredo J. Jarma1, 2, Juan C. Arbelaez ${ }^{2}$ y Jairo Clavijo² \\ Recibido para evaluación: Junio 15 de 2007 - Aceptado para publicación: Octubre 30 de 2007
}

\begin{abstract}
RESUMEN
Se condujeron estudios de laboratorio con el propósito de evaluar el efecto de los factores temperatura, luz, sustancias metabólicas y escarificación, sobre la germinación de la falsa caminadora (Ischaemum rugosum Salisb.). Las pruebas de germinación se adelantaron en cajas de germinación con agua destilada utilizando un diseño experimental completamente al azar con tres repeticiones. Los resultados indicaron que bajo las condiciones ambientales de la Sabana de Bogotá, la imbibición de las semillas de falsa caminadora presentan un período de reposo o "plateau" entre las 12 y 48 horas después de iniciarse la etapa de absorción de agua. Los porcentajes de germinación fueron afectados de manera significativa por temperaturas extremas (10 y $50{ }^{\circ} \mathrm{C}$ ), en tanto que la exposición a la luz rojo-lejano tuvo un efecto positivo sobre el número de semillas germinadas $(\operatorname{Pr}<0.05)$. La eliminación mecánica de la testa, mostró una significativa incidencia sobre la emergencia respecto a los tratamientos químicos de escarificación. La aplicación de giberelinas $\left(\mathrm{GA}_{3}\right)$ y Nitrato de Potasio $\left(\mathrm{KNO}_{3}\right)$ presentaron porcentajes de emergencia mayores con respecto a los demás factores evaluados. Con base en los resultados, se puede inferir que las semillas sexuales de falsa caminadora presentan la respuesta típica de una especie fotoblástica y se comprueba la importancia que tienen otros factores físicos, como la presencia o ausencia de la testa y el estímulo iniciador de sustancias metabólicas sobre la germinación de la misma.
\end{abstract}

Palabras clave: Falsa caminadora, malezas en arroz, fisiología de semillas, germinación de malezas.

\begin{abstract}
The effect of temperature, light, metabolic substances and scarification on the Ischaemum rugosum Salisb germination were evaluated. The germination tests were performed in germination boxes with distilled water using a completely randomized design with three replicates. The results indicated that the after the imbibition phase Ischaemum rugosum Salisb., presents a latency period or "plateau" about 12 to 48 hour after water adsorption is initiated. Germination percentages were affected significantly by extreme
\end{abstract}

'Universidad de Córdoba, Departamento de Ingeniería Agronómica y Desarrollo Rural, Carrera 6 No 76 - 103, Telefax (4) 7860255,Email: ajarma@sinu.unicordoba.edu.co

${ }^{2}$ Universidad Nacional de Colombia - Sede Bogotá 
temperatures $\left(10\right.$ and $50{ }^{\circ} \mathrm{C}$ ), the far-red light exposure had a positive effect on the number of germinated seeds $(\operatorname{Pr}<0.05)$. Scarification had a significant effect on emergency with respect to chemical treatment. Metabolic substances, such as gibberellins $\left(\mathrm{AG}_{3}\right)$ and Potassium Nitrate $\left(\mathrm{KNO}_{3}\right)$, showed higher emergency percentages. Based on the results, it can be inferred that Ischaemum rugosum Salisb. seeds present a typical photoblastic species behavior and the importance of physical factors, such as absence of the seed coat and the stimulus of metabolic substances on seed germination.

Key words: Wrinklegrass, weeds rice, seed physiology, weed germination.

\section{INTRODUCCIÓN}

Uno de los factores que tienen mayor relevancia dentro de la estructura de costos en la producción de arroz en Colombia es el control de arvenses. Dentro de estas, la falsa caminadora (Ischaemum rugosum Salisb.) puede representar hasta un 33\% de reducción en los rendimientos (Clavijo y Medina, 2004). I. rugosum es una gramínea de crecimiento anual, culmo decumbente con una altura de 10 a 100 $\mathrm{cm}$. Las hojas pueden llegar a tener de 5 a 30 $\mathrm{cm}$ de largo, en tanto que su ancho puede ser de 3-15 mm (Clayton et al., 2005). Su adaptación a diferentes habitats del trópico de América y Asia, tales como suelos inundados o secos, ha sido reportada por Baki (1992). Asimismo, Nabi (1999), indica que la falsa caminadora es una planta $\mathrm{C}_{4^{\prime}}$ y que ésta condición le ha permitido adaptarse a ambientes calurosos, secos, húmedos y de alta intensidad lumínica, especialmente en los trópicos. En Malasia, es considerada una de las arvenses de mayor competitividad, no sólo en cultivos de arroz, sino que además limita los rendimientos de manera importante en caña de azúcar y palma de aceite (Bakar y Ahmed, 2003). Los mismos autores informan que es una especie prolífica (alrededor de 18000 semillas por planta por año) y que esta producción puede prolongarse hasta por 10 semanas después de la aparición de la primera espiga. Baki (1986), sugiere que una alta capacidad numérica de reproducción no es garantía de la supervivencia y la colonización; sin embargo, la eficiencia de I. rogusum en la distribución de los asimilados hacia la semilla que puede ser del 40\% (Baki, 1988).

Adicional a su habilidad para adaptarse, ya se ha reportado la resistencia de $I$. rogusum a herbicidas del grupo D/22 (bipiridilos) en Malasia (Bakar, 2007) y en Colombia al grupo A/1 (inhibidores de la ACCasa) (Valverde, 2007), aunque en Venezuela, recientemente se ha reportado su susceptibilidad al herbicida halosulfuron-metil (Suárez et al., 2004).

Baskin y Baskin (2001b), indicaron que la germinación es una de las fases más críticas en el desarrollo de cualquier especie y que cada una de estas posee un rango específico de factores ambientales que son necesarias para que este proceso sea exitoso; entre estos, los más conocidos son temperatura, luz, $\mathrm{pH}$ y humedad del suelo. Trabajos desarrollados por Bakar y Ahmed (2003), indican que la semilla de $I$. rugosum se comporta como una especie fotoblástica (Berrie, 1987), ya que depende de la luz para la germinación. En este sentido, Hill et al. (1989), informan que la profundidad a la que se coloquen las semillas, juega un papel determinante en la germinación, ya que la pobre calidad de luz recibida por semillas enterradas, induce una dormancia forzada. En ese sentido, Torres y Guzmán (2003), encontraron que, después de $8 \mathrm{~cm}$ de profundidad, los porcentajes de germinación de esta especie se redujeron a cero. Pons (2000), indica que tanto la cantidad de luz [tiempo de exposición a la Densidad del Flujo de Fotones Fotosintéticos (DFFF)], como 
la calidad de la misma [especialmente la relación entre las longitudes del rojo y rojo-lejano $(R: R L)]$, son ejemplos de señales ambientales que intervienen de manera importante para el establecimiento y supervivencia de la semilla.

Estudios de germinación en I. rugosum respecto a factores como temperatura y sustancias metabólicas $\left(\mathrm{KNO}_{3}\right)$, han indicado que esta especie presenta los mayores porcentajes de germinación cuando se aplica el nitrato de potasio al medio y cuando la temperatura oscila entre 25 y $30{ }^{\circ} \mathrm{C}$ (Bakar y Ahmed, 2003). Con base en la importancia que tiene para el país el sector arrocero, y dentro de este, el manejo integrado de malezas, se planteó el presente trabajo, con el objetivo de identificar el o los factores que inciden en la germinación de la falsa caminadora, como un aporte al conocimiento de esta especie, para la implementación de posibles estrategias de manejo.

\section{MATERIALES Y MÉTODOS}

El trabajo se desarrolló en el segundo semestre de 2005 en el Laboratorio de Fisiología de Cultivos de la Facultad de Agronomía de la Universidad Nacional de Colombia, sede Bogotá, con semilla sexual de I. rugosum proveniente de plantas colectadas el mismo año en el lote 1-9 del C.I. Nataima de Corpoica, correspondiente a la Vereda San francisco en el Municipio del Espinal (Tolima). Las semillas fueron empacadas en bolsas de papel para su traslado, secadas y almacenadas bajo condiciones adecuadas durante un mes para homogenizar dormancia.

Se utilizó únicamente semilla sin cubierta (cariópside), tal como lo registra la figura 1. Con el propósito de reducir las fuentes de variación, de las dos semillas presentes en cada espiguilla, se tomó la unidad de mayor tamaño, cuyo peso de 1000 unidades estuvo estandarizado en promedio en $1.45 \mathrm{~g}$. Las pruebas de germinación se realizaron en cajas de Petri con papel absorbente, en las que se ubicaron 15 semillas por caja y un volumen de agua destilada de 5 $\mathrm{ml}$. En todos los casos, excepto el ensayo con temperaturas donde los tratamientos fueron diferenciales para este factor, se colocaron las cajas en bandejas de germinación sometidas a temperatura constante de $28^{\circ} \mathrm{C}$.

Los tratamientos fueron distribuidos utilizando un diseño experimental completamente al azar con arreglo factorial cuando éste fue necesario, donde los niveles de los factores dependieron de cada uno de los experimentos, lo cual se abordará más adelante. En todas las pruebas la variable respuesta fue el porcentaje de germinación. El criterio principal en la escogencia de los tratamientos se hizo con base en los resultados del trabajo adelantado por Clavijo et al. (2004), en el que, bajo condiciones
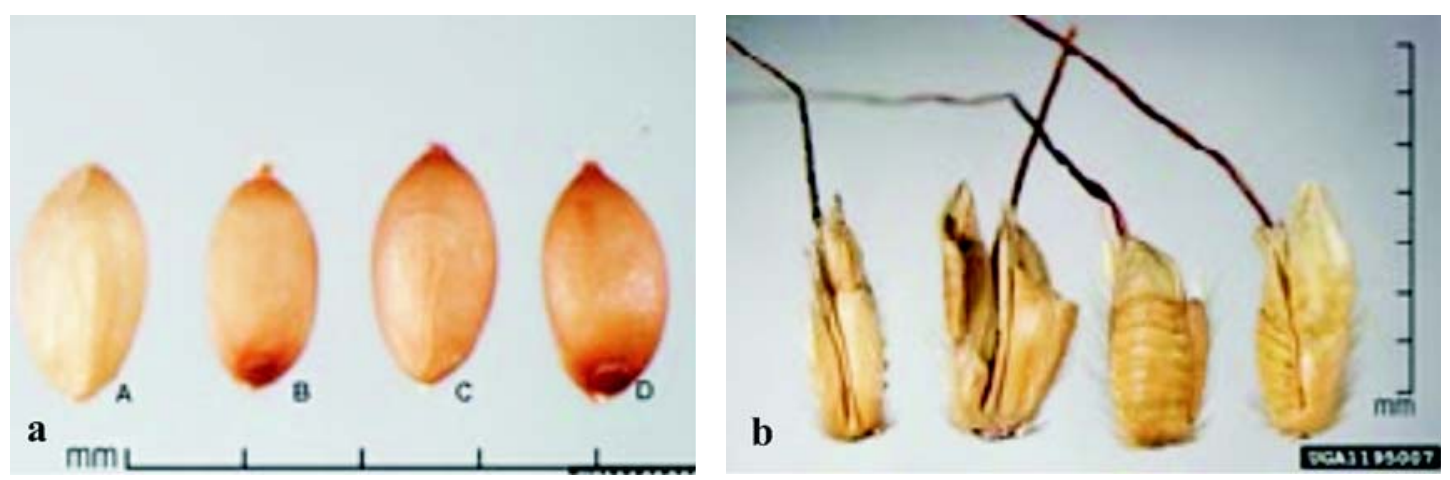

Figura 1. Semilla sexual de Ischaemum rugosum Salisb. a. Cariópside. b. Espiga. (Tomado de USDA, NRCS. Invasive and exotic species, 2005) 
similares los investigadores reportaron los efectos de cuatro factores sobre la germinación de la misma especie.

\section{Prueba estándar de germinación}

Esta prueba se realizó con el propósito de cuantificar el porcentaje de emergencia de la falsa caminadora al momento de iniciar la investigación, bajo condiciones de agua, luz y temperaturas estándar. Para esto, la prueba se realizó durante 20 días en una cámara de germinación con HR del 80\%, 12:12 horas de luz:oscuridad y temperatura controlada de $28{ }^{\circ} \mathrm{C}$, de acuerdo con resultados reportados por Bakar y Ahmed (2003) para esta especie. Se utilizaron 12 cajas de Petri con 25 semillas cada una, de las cuales, cada cuatro cajas (100 semillas) se consideraron una repetición; las lecturas se hicieron cada tres días determinando la germinación diaria como porcentaje (\%).

\section{Curva de imbibición}

Con el propósito de identificar las fases de imbibición, se colocaron 100 semillas distribuidas en cuatro cajas Petri, a las cuales se les tomó el peso fresco inicial en balanza de precisión y luego se les sometió a imbibición con $5 \mathrm{ml}$ de agua destilada en un germinador estandarizado a $28{ }^{\circ} \mathrm{C}$. Posteriormente, las lecturas de peso se realizaron a las $0.5,1,2,4$, $8,12,24,48,72$ y 120 horas. Los resultados se graficaron en función del tiempo.

\section{Niveles de luz}

Para este experimento se plantearon dos ensayos, cada uno bajo un diseño experimental completamente al azar con arreglo factorial de $3 \times 4$. En el primero de ellos, las semillas estuvieron expuestas a diferentes tipos de luz (rojo, rojo-lejano y oscuridad) durante 24 horas (factor A), y diferentes tiempos de imbibición ( 0 , 1,3 y 6 minutos), lo que se consideró como factor B. El segundo trabajo fue igual, sólo que el tiempo de exposición a cada tipo de luz fue de 48 horas. Para irradiar las semillas con los diferentes tipos de luz, se utilizó la infraestructura que para tal fin tiene la Facultad de Agronomía de la Universidad Nacional, donde, en un cuarto de luz controlada se ubicaron cámaras que contenían cuatro fuentes de luz blanca, fluorescente, fría, de 25 watts. A estos tubos se les colocó papel celofán de color rojo y/o azul hasta verificar una longitud de onda de $680 \mathrm{~nm}$ para la cámara del efecto del rojo (R), y de $730 \mathrm{~nm}$ para el efecto del rojo lejano (Fr). En otro recipiente totalmente sellado se colocaron las semillas del tratamiento de oscuridad y las lecturas se realizaron en el cuarto oscuro durante 10 días.

\section{Niveles de temperatura}

Para determinar el efecto de temperaturas altas y bajas sobre el porcentaje de germinación, se estableció un diseño experimental completamente al azar con un arreglo factorial de $3 \times 4$, donde el factor A correspondió a los grados de temperatura de exposición $(10,30$ y $50{ }^{\circ} \mathrm{C}$ ) y el factor $\mathrm{B}$ a los tiempos de exposición (4, 8, 12 y 24 horas). Luego de sometidas a cada tratamiento, las semillas se Ilevaron simultáneamente al germinador, cuyas especificaciones fueron comentadas anteriormente en la prueba estándar de germinación. Las lecturas se realizaron durante 10 días (240 horas).

\section{Sustancias metabólicas y reguladores del crecimiento}

Las semillas se sometieron a imbibición en diferentes sustancias metabólicas y reguladores de crecimiento. Para ello se usó un diseño experimental completamente al azar con ocho tratamientos que incluyen las sustancias individuales y algunas combinaciones (Tabla 1). El tratamiento que combina giberelina $\left(\mathrm{AG}_{3}\right)$, tiourea y nitrato de potasio $\left(\mathrm{KNO}_{3}\right)$, se consideró, conjuntamente con la imbibición en agua, como testigo por los excelentes resultados demostrados en esta especie en pruebas del Laboratorio de la Universidad que no están referenciadas. Diariamente, durante 10 días se hicieron las lecturas de germinación. 
Tabla 1. Tratamientos de sustancias metabólicas y reguladores del crecimiento aplicados a semillas de I. rugosum.

\begin{tabular}{ccc}
\hline Sustancia & Concentración & Tiempo imbibición (h) \\
\hline $\mathrm{AG}_{3}+$ Tiourea $+\mathrm{KNO}_{3}$ & $100 \mathrm{ppm}+1 \%+10 \%$ & 24 \\
Kinetina & $10 \mathrm{ppm}$ & 24 \\
$\mathrm{AG}_{3}$ & $100 \mathrm{ppm}$ & 24 \\
Kinetina $+\mathrm{AG}_{3}$ & $10 \mathrm{ppm}+100 \mathrm{ppm}$ & $12-12$, respectivamente \\
$\mathrm{AG}_{3}+$ Kinetina & $100 \mathrm{ppm}+10 \mathrm{ppm}$ & $12-12$, respectivamente \\
Tiourea & $1 \%$ & 24 \\
$\mathrm{KNO}_{3}$ & $10 \%$ & 24 \\
Agua destilada & & 24 \\
\hline
\end{tabular}

\section{Escarificación}

En este experimento se usaron métodos de escarificación mecánica y química. La escarificación mecánica consistió en la eliminación de la testa mediante frotes continuos con lija durante 5 minutos y punteadura de la testa con alfiler entomológico delgado, con el cuidado de no dañar el embrión. En los tratamientos de escarificación química se utilizó ácido sulfúrico $\left(\mathrm{H}_{2} \mathrm{SO}_{4}\right)$ en concentraciones de 100,50 y $25 \%$, combinado con tiempos de inmersión de 1, 2 y 4 minutos. Tal como se realizó para los trabajos anteriores, luego de los tratamientos, las semillas se colocaron en bandejas de germinación a $28{ }^{\circ} \mathrm{C}$ durante 10 días. El trabajo se estructuró bajo un diseño completamente al azar con 12 tratamientos (9 escarificaciones químicas de las combinaciones de concentración y tiempo con $\mathrm{H}_{2} \mathrm{SO}_{4^{\prime}}$ dos escarificaciones mecánicas y el testigo absoluto sin escarificar).

\section{RESULTADOS Y DISCUSIÓN}

\section{Prueba estándar de germinación}

La tabla 2 muestra los porcentajes de germinación registradas. Las primeras semillas iniciaron su proceso de germinación a partir de las 72 horas (3 días), y luego de 10 días se alcanzaron los valores máximos, los cuales se mantuvieron hasta el final de las mediciones (alrededor del 60\%).

\section{Curva de imbibición}

La figura 2 muestra la ganancia de peso de semillas de $I$. rugosum en función del tiempo, hasta 5 días después de iniciarse la imbibición. Azcón y Talón (2003) y Bewley y Black (1994), definen este proceso como la toma de agua por parte de los tejidos de reserva de la semilla, a una velocidad intermedia hasta completar su hidratación donde se estabiliza, sin importar su viabilidad. Si se consideran las tres fases de

Tabla 2. Porcentaje de germinación de I. rugosum en función del tiempo en cámara de germinación $\left(28{ }^{\circ} \mathrm{C}\right.$, 12 horas luz y HR $=80 \%$ ).

\begin{tabular}{cccccccc}
\hline & \multicolumn{7}{c}{ Días después de establecido } \\
\cline { 2 - 7 } & 1 & 2 & 3 & 5 & 10 & 15 & 20 \\
\hline $\begin{array}{c}\text { Porcentaje de } \\
\text { germinación (\%) }\end{array}$ & 0 & 0 & 21.6 & 60.5 & 62.7 & 63.3 & 63.8 \\
\hline
\end{tabular}




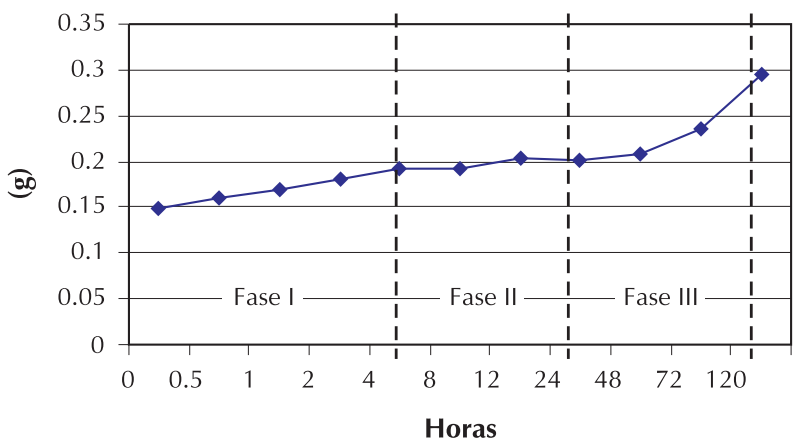

Figura 2. Ganancia de peso de 100 semillas de I. rugosum en función del tiempo (horas).

imbibición, podría ubicarse a la Fase I entre 0 y 4 horas, la Fase II entre 4 y 24 y la Fase III entre 24 y 120 . Esta tendencia de la imbibición puede considerarse como normal de acuerdo a las definiciones descritas anteriormente; se destaca que el período de reposo o "plateau" se presentó en la Fase II. Estos resultados tienen similitud a los reportados por Clavijo et al. (2004), en donde el "plateau" se presentó entre las 3.5 y 4.5 horas. Tendencias similares de la curva de imbibición reportan Moreno et al., (2006), en semillas sin testa al compararlas con semillas con testa en caucho (Hevea brasiliensis Muell.). Es importante resaltar que en el ensayo de Clavijo et al. (2004), la finalización del "plateau" se observó luego de 4.5 horas de imbibición, mientras que en el presente trabajo se alcanzó alrededor de las 20 horas. Lo anterior podría ser explicado por el tipo de semilla utilizado por los investigadores anteriormente citados, en las que la cubierta podría estar actuando como un absorberte y almacenador de agua permitiendo un proceso de imbibición más rápida.

\section{Niveles de luz}

La respuesta de las semillas de I. rugosum ante el estímulo de la luz fue linealmente positiva, en el sentido de observarse un aumento significativo en el porcentaje de germinación ante la presencia de luz, independientemente que esta fuera roja (R) o rojo-lejana (Fr) (Figura 3).

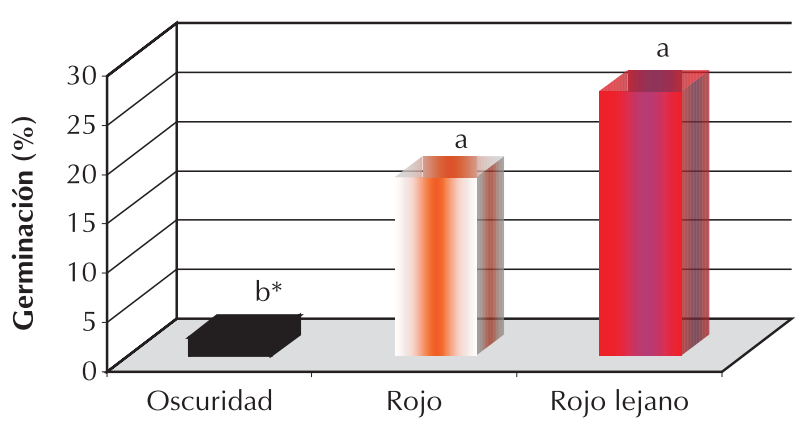

Figura 3. Porcentaje de germinación de I. rugosum ante estímulos lumínicos.

El análisis estadístico indicó que no se presentaron diferencias significativas $(P r>0.05)$ para ninguna de las interacciones de los factores considerados (tiempo de exposición a la luz, tipo de luz y tiempo de imbibición). Asimismo, no se detectaron diferencias estadísticas para los efectos individuales tiempo de exposición y tiempo de imbibición, pero estas sí fueron evidentes para el tipo de luz $(\operatorname{Pr}<0.05)$. Los resultados anteriores confirman lo reportado por varios investigadores quienes indican que $I$. rugosum es una especie fotoblástica al responder de manera positiva ante estímulos lumínicos (Berrie, 1987; Bakar y Ahmed, 2003; Torres y Guzmán 2003). Aunque los mayores porcentajes de emergencia no superaron el 30\%, estos valores, fueron significativamente mayores a los observados en el testigo (oscuridad), indicando el efecto positivo de la luz sobre la germinación de esta especie. Esto puede ser determinante en sistemas de cultivo donde la preparación del suelo permite la exposición de las semillas a la incidencia de la luz natural. Por otra parte, los resultados encontrados en el presente estudio son diferentes a los reportados por Clavijo et al. (2004), quienes no observaron ningún efecto de la luz roja (R) o azul sobre el porcentaje de emergencia de las semillas de $I$. rugosum. Es probable que, de manera similar a lo observado para la prueba de imbibición, la cubierta de las semillas utilizadas en el 2004, funja como una capa protectora que no permitió al cariópside recibir el estímulo de luz requerido para desencadenar el proceso de germinación, 
y que los resultados observados en el espectro del rojo (R), donde el rojo lejano (Fr) es inactivo fisiológicamente, sean consecuencia de una rápida interconversión de este al rojo lejano (Fr). Bakar y Ahmed (2003), sugieren que la baja o nula luminosidad que recibe la semilla enterrada a profundidades superiores a $2 \mathrm{~cm}$, inducen una dormancia en la especie que reduce significativamente los porcentajes de germinación. En este mismo sentido Rao y Moody (1995), en un trabajo de suelos saturados con diferentes láminas de agua, indicaron que las semillas de $I$. rugosum que flotaron en la superficie del agua germinaron en un $98 \%$ debido a las condiciones de luz y temperatura que fueron favorables.

\section{Niveles de temperatura}

El análisis de varianza indicó que se presentaron diferencias altamente significativas entre los niveles de temperatura $(\operatorname{Pr}<0.01)$ y significativas entre los tiempos de exposición $(\operatorname{Pr}<0.05)$, aunque no para la interacción de estos. Los resultados (Tabla 3) sugieren que la exposición a temperaturas extremas, principalmente las altas, afectaron de manera importante el porcentaje de germinación de

Tabla 3. Porcentaje de germinación de semillas de I rugosum ante tres niveles de temperatura y cuatro tiempos de exposición.

\begin{tabular}{cc}
\hline $\begin{array}{c}\text { Nivel de temperatura } \\
\left({ }^{\circ} \mathrm{C}\right)\end{array}$ & $\begin{array}{c}\text { Porcentaje de } \\
\text { germinación }\end{array}$ \\
\hline 10 & $25.0 \mathrm{~b}^{*}$ \\
30 & $41.9 \mathrm{a}$ \\
50 & $8.3 \mathrm{~b}$ \\
Tiempos de exposición & \\
(horas) & \\
4 & $30.3 \mathrm{a}$ \\
8 & $26.8 \mathrm{ab}$ \\
12 & $22.8 \mathrm{bc}$ \\
24 & $20.2 \mathrm{c}$ \\
\hline
\end{tabular}

* Promedios con la misma letra dentro de una columna son iguales estadísticamente de acuerdo al Test protegido de DMS a un nivel del 5\%.
I. rugosum, reduciéndolo hasta cinco veces, independientemente que estas hayan sido expuestas 4, 8, 12 o 24 horas. En la misma tabla se observa el efecto del tiempo de exposición a las diferentes temperaturas; se encontró que en la medida que este tiempo fue superior, es decir, mayor tiempo de exposición, el porcentaje de germinación se redujo, aunque no de manera tan concluyente como se observó para el efecto de la temperatura. Para efectos de conveniencia en la interpretación de los resultados, puede asumirse que estos valores denotarían mayor importancia a temperatura alta, donde los porcentajes de germinación pueden reducirse significativamente si estas llegan a ser constantes hasta por 12 a 24 horas.

El efecto de la temperatura sobre la germinación de I. rugosum fue reportado por Bakar y Ahmed (2003) quienes encontraron que el porcentaje de germinación fue creciente en la medida que la temperatura de incubación fue aumentando, hasta un punto de germinación máxima (entre 75 - 90\% a temperaturas constantes que oscilaron entre 25 y $30{ }^{\circ} \mathrm{C}$ ), para luego decrecer hasta un $30 \%$ por efecto de las temperaturas elevadas $\left(40^{\circ} \mathrm{C}\right)$. Es importante resaltar que, en su hábitat natural, las semillas están expuestas a temperaturas alternas y no constantes, por lo que, usualmente estas temperaturas son más favorables para la germinación que las constantes (Baskin y Baskin, 2001a), aunque en la cámara de germinación utilizada en el presente trabajo, no fue posible hacer esta programación. La temperatura máxima óptima para la germinación depende de la especie y que puede variar desde $22-25{ }^{\circ} \mathrm{C}$ en Apium graveolens hasta $45{ }^{\circ} \mathrm{C}$ en Sorghum halepense; adicionalmente, exposiciones a temperaturas elevadas, pueden inducir una especie de dormancia (Baskin y Baskin, 2001a).

\section{Sustancias metabólicas y reguladores del crecimiento}

Se observaron altos porcentajes de germinación en presencia de las sustancias metabólicas 
aplicadas y, para todos los tratamientos, estos porcentajes fueron significativamente superiores respecto al tratamiento con agua $(\operatorname{Pr}<0.05)$. Las pruebas estadísticas determinaron que entre los tratamientos que mayor porcentaje de germinación mostraron, es decir nitrato de potasio, kinetina, tiourea, ácido giberélico + kinetina y kinetina + ácido giberélico, no se presentaron diferencias significativas entre sí (Tabla 4). La aplicación de $\mathrm{KNO}_{3}$ estimuló la germinación en un $96 \%$, siendo mayor que el testigo utilizado en el laboratorio $\left(\mathrm{AG}_{3}+\right.$ Tiorea $\left.+\mathrm{KNO}_{3}\right)$ y que el efecto individual de la giberelina. Clavijo et al. (2004), no encontraron repuestas al nitrato de potasio ni a la tiourea, sustancias que registraron altos porcentajes de germinación en el presente estudio. Es probable que en el citado estudio, las concentraciones utilizadas no fueron las adecuadas para estimular el proceso, o que no existió coincidencia entre el mecanismo sobre el cual actúan y la época de aplicación. Sin embargo, la respuesta a sustancias hormonales reportada en el mismo estudio mostró valores similares a los encontrados en el presente trabajo, indicando que aplicaciones de kinetina (citoquinina), ácido giberélico (giberelina) o efectos combinados pueden estimular de manera importante la germinación de $I$.

Tabla 4. Porcentaje de germinación de I. rugosum ante la aplicación de hormonas y sustancias metabólicas.

\begin{tabular}{cc}
\hline Tratamiento & Germinación (\%) \\
\hline $\mathrm{KNO}_{3}$ & $96 \mathrm{a}^{*}$ \\
Tiourea & $86 \mathrm{ab}$ \\
$\mathrm{AG}_{3}+$ Kinetina & $83 \mathrm{ab}$ \\
Kinetina & $83 \mathrm{ab}$ \\
Kinetina $^{-\mathrm{AG}_{3}}$ & $83 \mathrm{ab}$ \\
$\mathrm{AG}_{3}+$ Tiourea $_{\mathrm{KNO}}$ & $73 \mathrm{~b}$ \\
$\mathrm{AG}_{3}$ & $66 \mathrm{~b}$ \\
Testigo (agua) & $20 \mathrm{c}$ \\
\hline
\end{tabular}

* Promedios con la misma letra son iguales estadísticamente de acuerdo al Test protegido de DMS a un nivel del $5 \%$. rugosum. Cohn (1987), reporta que aplicaciones de giberelinas, citoquininas y etileno actuando de manera simple o combinada pueden romper la dormancia de muchas semillas y que el ácido abscísico puede inhibir la germinación. En este sentido, Salisbury (2000) informa que entre los tratamientos químicos empleados para mejorar la germinación están algunos reguladores de crecimiento como el ácido giberélico, que además interviene en la elongación y emergencia de la radícula a través del endospermo, la cubierta seminal o la cubierta del fruto que restringen su crecimiento. El autor indica que con el uso de estas fitohormonas se ha comprobado que se superan los períodos de latencia que se presentan en las semillas de algunas especies actuando como sustitutos de bajas temperaturas, días largos o luz roja.

Respuestas similares a las encontradas en el presente trabajo con nitrato de potasio y tiourea, fueron reportados por Suárez (1983) en pasto carimagua (Andropogon gayanus Kunth) y Bakar y Ahmed (2003), en I. rugosum, los cuales atribuyeron esta respuesta al efecto combinado del nitrato y la luz sobre el rompimiento de la dormancia de las semillas. Cohn y colaboradores (1983), en trabajos adelantados con arroz rojo, indicaron que las respuestas a la aplicación de nitritos se dio solamente bajo unos rangos de temperatura $\left(25-30{ }^{\circ} \mathrm{C}\right)$, y que, la germinación se redujo de manera importante cuando la temperatura estuvo por debajo de $20{ }^{\circ} \mathrm{C}$.

El papel que juegan las hormonas sobre la germinación de malezas en diversos cultivos, es uno de los aspectos estratégicos que se deben considerar ya que, debido a la gran cantidad de herbicidas que se utilizan en agricultura, es razonable preguntarse si estos compuestos, hormonales o no, tienen algún efecto sobre la germinación. Baskin y Baskin (2001b) indican que dependiendo del tipo de herbicida y la especie, la germinación puede ser incrementada, inhibida o no afectada. 


\section{Escarificación}

En razón a que los porcentajes de emergencia de I. rugosum tuvieron valores de cero para los tratamientos con ácido sulfúrico cuando se trataron por 2 y 4 minutos, sólo se comparará el efecto de este tratamiento químico con los demás, cuando se trataron las semillas por espacio de un minuto. La figura 4 muestra el porcentaje de germinación de las semillas sometidas a los tratamientos de escarificación. Los resultados indican que las semillas tratadas con ácido sulfúrico, a cualquiera de las concentraciones estudiadas, redujeron significativamente el porcentaje de germinación respecto a las no tratadas químicamente. Lo anterior probablemente se deba al daño que este ácido causó en el embrión de las semillas, que se presentaba descubierto, como se mostró en la figura 1a. Por otra parte, la eliminación de la testa del cariópside mediante raspadura mecánica, estimuló significativamente la germinación de las semillas en comparación con los demás tratamientos, confirmando que para la especie, esta capa de células juega un papel definitivo, no sólo a nivel de su función como impermeabilidad a agua y $\mathrm{O}_{2}$ en diferentes grados, si no como una barrera física, que, aunque no del todo limitante para el desarrollo del embrión, si retarda el tiempo de la emergencia de este. Esta barrera impide que

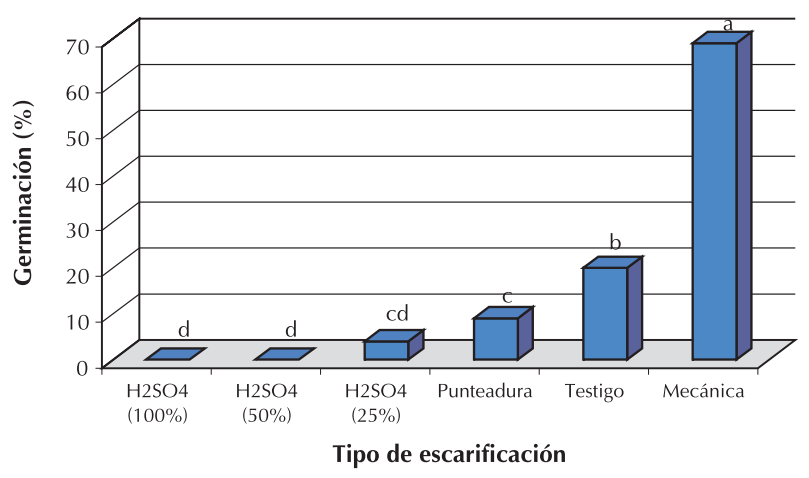

Figura 4. Porcentaje de germinación de I. rugosum ante diversos tratamientos de escarificación. (*Barras con la misma letra son iguales estadísticamente de acuerdo al Test protegido de DMS a un nivel del 5\%). la citrato oxidasa esté en permanente trabajo lo cual implicaría una inminente germinación; asimismo, impide que una reacción de oxidación inicie la germinación, estableciendo una baja tensión de $\mathrm{O}_{2}$, por lo que tiene la responsabilidad de mantener bajos niveles de respiración, para mantener viva la semilla pero con un metabolismo muy lento. Otros aspectos importantes que podrían estar explicando la respuesta de I. rugosum en ausencia de la testa, son los relacionados con la toma del agua, difusión de inhibidores o la captación de la luz. La ausencia de esta, puede facilitar el movimiento libre de permeasas e hidrolasas implicadas en el inicio del proceso de germinación, permitir el lavado de inhibidores metabólicos asociados al embrión y/o, facilitar el efecto de la luz sobre el embrión para estimular el proceso. Los resultados observados en este trabajo son coincidentes con los de Clavijo et al. (2004), en los que se reporta un efecto positivo sobre el porcentaje de germinación con escarificación mecánica; sin embargo, en dicho trabajo, el efecto del ácido sulfúrico fue menos inhibidor que lo encontrado en el presente estudio, lo que podría explicarse por el tiempo utilizado en la inmersión en cada caso (30 segundos y 1 minuto, respectivamente).

\section{CONCLUSIONES}

- El comportamiento de las semillas de Ischaemum rugosum ante los estímulos de luz roja y roja-lejana comparados con los efectos de la oscuridad, indican que es una especie fotoblástica positiva y posiblemente una rápida conversión del rojo al rojo lejano.

- Los extremos de temperatura afectan negativamente los porcentajes de germinación, aunque los efectos de las temperaturas elevadas son más decisivos que los de temperaturas bajas, encontrándose los porcentajes de germinación más altos en rangos intermedios $\left(30{ }^{\circ} \mathrm{C}\right)$. 
- La germinación de Ischaemum rugosum se vio favorecida por el efecto de algunas sustancias metabólicas como el $\mathrm{KNO}_{3}$ y otras hormonales como citoquininas, giberelinas y su combinación.
- La presencia o ausencia de la testa juega un papel definitivo sobre la germinación de las semillas, siendo ésta estimulada de manera importante cuando esta capa de células está ausente e inhibida en su ausencia.

\section{BIBLIOGRAFÍA}

Azcón-Bieto, J. y M, Talón. 2003. Fundamentos de Fisiología Vegetal. McGraw-Hill Interamericana, Ciudad de edición? 450 p.

Bakar, B. 2007. Bipyridiliums (group D/22) resistant saramollagrass (Ischaemum rugosum) Malaysia. http:// www.weedscience.org/Case/ Case.asp?ResistID=139. [Accedido: 06-04-07]

Bakar, B. y Ahmed, L. 2003. Seed germination, seedling establishment and growth patterns of wrinklegrass (Ischaemum rugosum Salisb.) Weed Biology and Management 3:8-14

Baki, B. 1986. The population biology of Oxalis corniculata L. Tesis, Ph.D, University of Wales, Bangor

Baki, B. 1988. The seed population dynamics of barnyardgrass [Echincohloa crusgalli (L.) P. Beauv.]. Proceedings of the National Seminar on Rice Weed Management (Penang, Malaysia 7-9 Junio, 1988). Malaysian Agricultural Research and Development Institute, Serdang, 299-314.

Baki, B. 1992. Ischaemum rugosum Salisb. En: Mannetje L. y Jones R. (Ed.). Plant Resources of South-East Asia, Forages. Pudoc Scientific Publishers, Wageningen, 146-148.
Baskin, C. y Baskin, J. 2001a. Constant versus alternating temperature regimes. Seeds. Ecology, Biogeography and Evolution of Dormancy and Germination. Academic Press, California, p12-13

Baskin, C. y Baskin, J. 2001b. Environmental factors causing changes in dormancy states. Seeds. Ecology, Biogeography and evolution of dormancy and germination. Academic Press. California -USA. 666 p. pp. 73-76.

Berrie, A. 1987. Plant Physiology. English Language Book Society, Longmans, Londres, 440-468.

Bewley, J. y Black, M. 1994. Seeds: Physiology of development and germination. Plenum Press, New York, 445p.

Clavijo, J. y Medina, H. 2004. Impacto socioeconómico de las malezas en el cultivo de arroz en Colombia. Revista Comalfi 31(1):13-23

Clavijo, J.; Montenegro, L.; Medina, J.; Barragán, E. y Duarte, C. 2004. Determinación de la inhibición y de la influencia de cuatro factores en la germinación de falsa caminadora Ischaemum rugosum Salisb. Revista Arroz 52(451):4-12 
Clayton, W.; Harman, K. y Williamson, H. 2005. Ischaemum rugosum. Copyright The Board of Trustees, Royal Botanic Gardens, Kew. En: World Grass Species - Ischaemum rugosum Description. http://www.kew.org/data/ grasses-db/www/imp05784.htm [Accedido: 09-28-2006]

Cohn, M.; Butera, D. y Hughes, J. 1983. Seed dormancy in Red Rice. III. Response to nitrite, nitrate and ammonium ions. Plant Physiology 73:381-384

Cohn, M. 1987. Mechanisms of physiological seed dormancy. En: Frasier,G. y Evans R. (Ed). Seed and seedbed ecology of rangeland plants. USDAARS, Washington, D.C. $311 \mathrm{p}$

Hill, N.M.; Patriquin, D. y Vander-Kloet, S. 1989. Weed seed bank and vegetation at the beginning and end of the first cycle of a four-course crop rotation with minimal weed control. Journal of Applied Ecology. 26: 233-246.

Moreno, F.; Plaza, G. y Magnitskiy, S. 2006. Efecto de la testa sobre la germinación de semillas de caucho (Hevea brasiliensis Muell.). Agronomía Colombiana 24(2):290-295

Nabi, L. 1999. The population biology of wrinklegrass (Ischaemum rugosum Salisb.). Tesis Ph. D., University of Malaya, Kuala Lumpur

Pons, T. 2000. Seed responses to light. En: Fenner, M (Ed). Seeds: The Ecology of Regeneration in Plant Communities. CAB International, New York, NY

Rao, A. y Moody, K. 1995. Effect of seeding depth and soil moisture regimes on emergence of Ischaemum rugosum Salisb. and Echinochloa glabrescens. Proceedings of the 15th Asian-Pacific Weed Science Society Conference, Asian-Pacific Weed Science Society Publication, 520-522

Salisbury, F. y Ross, C. 2000. Fisiología de las Plantas. Editorial Paraninfo Thomson Learning, Madrid, 988p.

Suárez, C. 1983. Estudio de algunos factores fisiológicos en germinación de pasto carimagua (Andropogon gayanus Kunth.). Tesis M. Sc. Universidad Nacional de Colombia, Bogotá

Suárez, L.; Anzalone, A. y Moreno, O. 2004. Evaluación del herbicida halosulfuronmetil para el control de malezas en el cultivo de arroz (Oryza sativa L.). Bioagro 16(3)

Torres, J. y Guzmán, P. 2003. Morfología de plántulas y fenología de algunas malezas gramíneas de importancia en campos de arroz del Norte de Tolima. Arroz. 51 (446):14

USDA - NRCS. 2005. Murainagrass. Identification, Biology, Control and Management Resources. http:// www.lucidcentral.org/keys/FNW/ F NW\% 20 grasses/ht m I/ f a c t $\%$ 2 00 s h e e t $\mathrm{s} /$ I schaemum\% 20 rugosum.htm [Accedido: 09-28-2006]

Valverde, B. 2007. ACCase inhibitors (group $\mathrm{A} / 1$ ) resistant saramollagrass (Ischaemum rugosum) http:// www.weedscience.org/Case/ Case.asp?ResistID=5143. [Accedido: 06-04-07] 